\title{
Prognostic and assessment value of hyperglycemia and glycosylated hemoglobin in critical patients
}

\author{
Z.L. Zhang1, X.M. Che ${ }^{2}$, Z.H. Bai ${ }^{1}$, W.J. Bu ${ }^{1}$, L. Bai ${ }^{1}$ and H.H. Pei ${ }^{1}$ \\ ${ }^{1}$ Emergency Department, Second Affiliated Hospital, Xi' an Jiaotong University, \\ Xi'an, Shaanxi Province, China \\ ${ }^{2}$ Department of General Surgery, First Affiliated Hospital, Xi'an Jiaotong University, \\ Xi'an, Shaanxi Province, China \\ Corresponding author: H.H. Pei \\ E-mail: honghongpei@yeah.net
}

Genet. Mol. Res. 13 (3): 7006-7012 (2014)

Received May 15, 2013

Accepted September 2, 2013

Published January 24, 2014

DOI http://dx.doi.org/10.4238/2014.January.24.8

\begin{abstract}
Hyperglycemia is common in critical patients and high blood glucose levels have a negative effect on their prognosis. The aim of this study was to investigate the effect of hyperglycemia and glycosylated hemoglobin $(\mathrm{GHb})$ in critical patients. A total of 648 critical patients were enrolled in the study and received a random blood glucose test when they entered the emergency department. If blood glucose was more than $11.1 \mathrm{mM}$, a GHb test was followed within 24 h. All patients were followed up for 28 days. According to diabetes mellitus (DM) history, GHb value, and outcome of follow-up, patients were divided into different groups, and mortality rates were calculated, respectively. Hyperglycemia was found in 67.44\% (437/648) of patients, and 51.49\% (225/437) and 48.51\% (212/437) had normal and elevated GHb levels, respectively. At the end of the follow-up period, 14 of the normal $\mathrm{GHb}$ patients and 32 of the elevated $\mathrm{GHb}$ patients died (6.22 and $15.09 \%$, respectively). In the normal GHb group, 53 had a DM history, 23 were newly diagnosed with DM, and 149 had hospital-
\end{abstract}


related hyperglycemia $(\mathrm{HRH})$; the mortality rates were $11.32 \%(6 / 53)$, $8.70 \%(2 / 23)$, and $4.03 \%(6 / 149)$, respectively. In the elevated $\mathrm{GHb}$ group, 114 had a DM history, 83 were newly diagnosed with DM, and 15 had $\mathrm{HRH}$; the mortality rates were $13.16 \%(15 / 114), 19.27 \%$ (16/83), and 6.67\% (1/15), respectively. Hyperglycemia and $\mathrm{GHb}$ might play important roles in the prognosis and assessment for critical patients, and the prognosis would vary according to the different causes of hyperglycemia.

Key words: Hyperglycemia; Glycosylated hemoglobin; Hospital-related hyperglycemia; Diabetes mellitus

\section{INTRODUCTION}

Hyperglycemia is common in hospitalized patients, and is generally associated with adverse outcomes (Capes et al., 2000). In our emergency department, especially in emergency intensive care units, more patients were found with hyperglycemia, regardless of whether or not they had a history of diabetes mellitus (DM). It is generally considered that hyperglycemia is most often seen in patients with a DM history, and that such patients would display a worse prognosis when hospitalized compared to those without DM(Guvener et al., 2002). However, cases of new onset hyperglycemia without DM history in-hospital have been increasing, and these patients also suffered higher risks for increased in-hospital morbidity and mortality compared to those without hyperglycemia (Bolk et al., 2001; Norhammar et al., 2002; Umpierrez et al., 2002).

It is well known that patients with high blood glucose levels suffer poorer outcomes. However, would critical patients with hyperglycemia suffer the same outcome? Which factors would be the most important to consider? What should be the next course of action? Our objective of this study was to answer these questions by performing a retrospective analysis in the hopes that the results of our study might provide more support to our proposal.

\section{MATERIAL AND METHODS}

\section{Patients}

A total of 648 critical patients (APACHE II score $>25$ ) were enrolled in this study between November 2009 and April 2011. The following patients were excluded: under 18 years of age, pregnant, blood diseased, diagnosed with hyperthyroidism, gastrointestinal bleeding, cancer, or uremia, surviving no longer than $24 \mathrm{~h}$ after cardiopulmonary resuscitation, physically injured, or chemically poisoned (Knaus et al., 1985). Of the 648 patients, 347 were male and 301 were female, and the mean age was $65.43 \pm 11.21$ years. The patients suffered from various different diseases such as acute pancreatitis $(4,0.62 \% ; 2$ of them received operations), diabetic ketoacidosis $(11,1.70 \%)$, diabetic hyperosmolar coma $(6,0.93 \%)$, acute obstructive suppurative cholangitis $(2,0.31 \%$; all received operations), acute coronary syndrome (146, $22.53 \%)$, severe dysrhythmia $(25,3.86 \%)$, acute heart failure $(98,15.12 \%)$, post cardiopulmonary resuscitation $(17,2.62 \%)$, severe pneumonia or unexplained infection $(119,18.36 \%)$, 
acute exacerbation of chronic bronchitis $(107,16.51 \%)$, and acute cerebrovascular disease (113, 17.44\%; 12 received operations).

\section{Experimental methods}

The fast random blood glucose test was instantly conducted on each of the 648 cases when they were admitted to the emergency department, and hyperglycemia was found in 437 of them based on the criterion of blood glucose $\geq 11.1 \mathrm{mM}$ (McCowen et al., 2001). All patients received a whole blood glycosylated hemoglobin $(\mathrm{GHb})$ test in the first $24 \mathrm{~h}$ after admission, and all relevant data, including age, gender, history (especially concerning DM history and its regular treatment), were collected. During in-patient treatment, blood glucose levels were maintained between 8-10 mM (Hoshino et al., 2010). Follow-up over 28 days was conducted through telephone or visiting. Information related to the DM diagnosis, including those with a DM history, those newly diagnosed by an endocrine specialist, and those whose diagnoses were unclear at the end of the study (recorded as non-DM), and the number of deaths were recorded. According to the follow-up data and the GHb values, the 437 hyperglycemia cases were divided into a history of DM group, a newly diagnosed DM group, and a hospital-related hyperglycemia (HRH) group. Each group was further divided into two subgroups according to their $\mathrm{GHb}$ values $(\mathrm{GHb}<7 \%=$ normal and $\mathrm{GHb} \geq 7 \%=$ abnormal). Mortality rates were calculated respectively in each group and subgroup (Table 1).

Table 1. Number of 28 day-death and different GHb in 437 random hyperglycemia.

\begin{tabular}{lccc}
\hline Group & Previously DM & Newly DM & HRH \\
\hline Normal GHb & 53 & 23 & 149 \\
& $(6)$ & $(2)$ & $(6)$ \\
Elevated GHb & 114 & 83 & 15 \\
& $(15)$ & $(16)$ & $(1)$ \\
Total & 167 & 106 & 164 \\
\hline
\end{tabular}

\section{Statistical analysis}

All data were analyzed with SPSS16.0. Measurement data are reported as means \pm standard deviation, and count data are used to describe mortality. The $\chi^{2}$ test was used for comparisons among groups, and $\mathrm{P}<0.05$ was considered to be a statistically significant difference.

\section{RESULTS}

Of the 648 critical patients meeting the criteria for enrollment in the study, 437 were found to suffer from random hyperglycemia. Of the 437 hyperglycemic patients, 225 patients (123 male and 102 female, aged $62.57 \pm 13.83$ years) had normal GHb levels, 14 of which died before the end of the 28-day follow-up period (accounting for 6.22\%). The other 212 hyperglycemic patients (117 male and 95 female, aged $67.78 \pm 14.66$ years) had elevated $\mathrm{GHb}$ levels, and 32 of them died before the end of the 28-day follow-up period (accounting for $15.09 \%)$. There was no statistically significant difference in the average age or gender composition between these two groups $(\mathrm{P}>0.05)$, but mortality rates did differ significantly $(\mathrm{P}<$ 
$0.01)$. Of the 437 hyperglycemic cases, 167 had a history of DM (38.22\% of hyperglycemic patients; $25.77 \%$ of all patients), 106 were given a new DM diagnosis (24.26\% of hyperglycemic patients; $16.36 \%$ of all patients), and 164 were diagnosed with HRH (37.52\% of hyperglycemic patients; $25.31 \%$ of all patients).

The mortality rates over the 28-day follow-up period were compared among groups according to the $\mathrm{GHb}$ value (Table 1). Mortality was significantly higher in the elevated $\mathrm{GHb}$ group than that in the normal $\mathrm{GHb}$ group $(\mathrm{P}<0.05)$. In normal $\mathrm{GHb}$ cases, those who had a history of DM suffered the highest mortality $(11.32 \%)$, while those with HRH suffered the lowest mortality $(4.03 \%)$; there was a significant difference in mortality among these three sub-groups $(\mathrm{P}<0.05)$. In elevated $\mathrm{GHb}$ cases, those newly diagnosed with DM suffered the highest mortality (19.27\%), while those with HRH suffered the lowest mortality (6.67\%); there was also a significant difference in mortality among these three sub-groups $(\mathrm{P}<0.01)$.

\section{DISCUSSION}

Regulated by the neuroendocrine system, energy metabolism for critical patients in acute exacerbation will change through the effect of hormones, interference of cytokines, resistance of insulin, and by other means (Grimble, 2002; Marette, 2002; Albrecht et al., 2004; Leonidou et al., 2007). In such situations, high blood glucose levels and high catabolism are commonly observed. This type of blood glucose increase is referred to as stress hyperglycemia, which usually occurs in three types of patients: those with a history of DM, no DM history but newly diagnosed with DM, and those with HRH. HRH is defined when patients' fasting blood glucose increased $(\geq 6.99 \mathrm{mM})$ or random blood glucose increased $(\geq 11.10 \mathrm{mM})$ once referred to the hospital, but recovered to normal blood glucose levels following discharge. High blood glucose levels could adversely affect the body and its functions in various ways: 1) decrease the condition of the internal environment through osmotic diuresis, especially original electrolyte disorders; 2) accelerate the occurrence of multiple organ dysfunction, as many organs (heart, brain, liver, kidney, etc.) become damaged through lactic acidosis, increase of NO, endothelial cell injury, and so on; 3) exacerbate the inflammation and endothelial injury by taking part in the systematic inflammatory response and further disrupting the balance between inflammation and pro-inflammation; 4) damage the immune system by inhibiting the function of white blood cells and the complement activity (McMahon and Bistrian, 1995; Cosio et al., 2005; de Rekeneire et al., 2006). Therefore, the blood glucose level plays an important role in the status assessment for critical patients, and is one of the independent hazardous factors that affect their prognosis.

The occurrence of stress hyperglycemia in critical patients is associated with various factors. For those with a history of DM, irregular therapies or inappropriate diets would cause high blood glucose, especially under stressful conditions. This might also occur in HRH cases and in those with "latent diabetes," that is, patients who did not have a recorded history of DM, but were newly diagnosed with DM during our treatment course. Stress hyperglycemia is very common in critical patients. In this study, 67.44\% (437/648) of critical patents suffered from stress hyperglycemia, which suggested that it was important to monitor the blood glucose level of critical patients. GHb is one of the important clinical indicators for diagnosing DM. It reflects the condition of blood control in the most recent 4 to 12 weeks, which, when combined with immediate blood glucose levels, would yield a greater clinical significance and could be 
one of the most important indicators in DM diagnoses (Selvin et al., 2004). In this study, 197 of the 212 patients with elevated GHb levels were ultimately diagnosed with DM (accounting for $92.92 \%$ ), suggesting that $\mathrm{GHb}$ is a very sensitive marker for DM diagnosis. Among the 270 random hyperglycemia sufferers with no DM history, 172 showed normal GHb levels, and 23 were ultimately diagnosed with DM, accounting for $13.37 \%$. Elevated GHb levels were observed in the remaining 98 critical patients, 83 of whom were ultimately diagnosed with DM, accounting for $84.69 \%$. In all 437 patients with random high blood glucose levels, the 28-day follow-up mortality was significantly higher in the elevated $\mathrm{GHb}$ group $(15.09 \%, 32 / 212)$ than in the normal GHb group $(6.22 \%, 14 / 225)(\mathrm{P}<0.01)$. This result suggested that it was beneficial to disclose and diagnose DM early by detecting the GHb levels for critical patients, and also suggested that patients with elevated GHb levels would suffer worse prognoses than those with normal GHb levels. This may result from the direct hazards and increased possibilities of wound and lung infections caused by high blood glucose.

In this study, patients with different GHb levels had different prognoses. For cases with normal GHb levels, those with a history of DM suffered the highest mortality, whereas those with HRH suffered the lowest. This is possibly owing to the fact that those with a DM history might have controlled their blood glucose levels well more recently, and their longterm DM condition resulted in their organs being overworked, thus causing further damage, which might have affected the prognosis. This might be the reason explaining why patients with a history of DM suffered the worst prognosis among all patients with normal GHb levels. For those with elevated GHb levels, newly diagnosed DM patients suffered the highest mortality, whereas the HRH patients suffered the lowest. Because the newly diagnosed DM patients were unaware of their blood glucose conditions, and therefore took no appropriate measures to control it, they were more likely to suffer from high blood glucose poisoning over a longer time period. Once they suffered from acute disease, they could not tolerate the further damage, and ultimately suffered from a poor prognosis. By contrast, because patients with a history of DM were fully aware of their own condition, they received effective therapies, and had better prognoses, even though they also suffered from relatively high blood glucose levels. Therefore, newly diagnosed DM patients would benefit more from increased attention and intervention in controlling blood glucose levels. The incidence of elevated $\mathrm{GHb}$ levels was higher in newly diagnosed DM patients $(78.30 \%, 83 / 106)$ than that in the HRH patients $(9.15 \%$, $15 / 164$ ), and regardless of the GHb value, the mortality of the HRH group was relatively low. Therefore, it was very important to detect the GHb value in patients who had no DM history but showed random high blood glucose levels. In addition, in order to improve the prognosis, patients with elevated GHb levels should be treated in the same way as those with DM with respect to receiving blood glucose control treatment.

For critical patients with hyperglycemia, insulin therapies might be the most effective way to control the blood glucose level. By controlling blood glucose levels, body metabolism might be adjusted, which would be of benefit (van den Berghe et al., 2001). Other therapy programs, such as $\mathrm{C}$ peptide, corticotrophin releasing factor, and so on, might also prove to be beneficial in the future (Hager et al., 2004; Kimura, 2005).

In our study, we concluded that hyperglycemia and GHb might play important roles in the prognosis and assessment of critical patients, and that the prognosis would vary according to the specific reasons contributing to the hyperglycemia. Therefore, random blood glucose levels should be tested for all critical patients, and should be performed as soon as possible 
in the emergency department. The GHb test should be followed if the blood glucose value is abnormal, and blood glucose control should be followed in-hospital. Our data may add to the evidence supporting these views.

There are some limitations of the study. We conducted a 28-day follow-up in all patients enrolled in our study, and considered those patients whose DM diagnoses were still unclear at the end of the study as non-DM patients. Therefore, the study might have been more complete if we had prolonged the follow-up time. We here demonstrated the potential value of testing for hyperglycemia and $\mathrm{GHb}$ levels in critical patients by analyzing the data of outcome and history of DM. However, we neglected the effect of some other factors, such as cardiovascular factors, pulmonary factors, and so on, which might also affect the prognosis (Nakamura et al., 2009; Ichiki et al., 2010). Furthermore, the exact mechanism underlying the effects of hyperglycemia and elevated GHb levels on the prognosis of different patients remains unknown. The potential value of other factors will be addressed in future studies.

\section{REFERENCES}

Albrecht C, Simon-Vermot I, Elliott JI, Higgins CF, et al. (2004). Leukocyte ABCA1 gene expression is associated with fasting glucose concentration in normoglycemic men. Metabolism 53: 17-21.

Bolk J, van der Ploeg T, Cornel JH, Arnold AE, et al. (2001). Impaired glucose metabolism predicts mortality after a myocardial infarction. Int. J. Cardiol. 79: 207-214.

Capes SE, Hunt D, Malmberg K and Gerstein HC (2000). Stress hyperglycaemia and increased risk of death after myocardial infarction in patients with and without diabetes: a systematic overview. Lancet 355: 773-778.

Cosio FG, Kudva Y, van der Verde M, Larson TS, et al. (2005). New onset hyperglycemia and diabetes are associated with increased cardiovascular risk after kidney transplantation. Kidney Int. 67: 2415-2421.

de Rekeneire N, Peila R, Ding J, Colbert LH, et al. (2006). Diabetes, hyperglycemia, and inflammation in older individuals: the health, aging and body composition study. Diabetes Care 29: 1902-1908.

Grimble RF (2002). Inflammatory status and insulin resistance. Curr. Opin. Clin. Nutr. Metab. Care 5: 551-559.

Guvener M, Pasaoglu I, Demircin M and Oc M (2002). Perioperative hyperglycemia is a strong correlate of postoperative infection in type II diabetic patients after coronary artery bypass grafting. Endocr. J. 49: 531-537.

Hager P, Hagman B, Wikstrom AC and Strommer L (2004). CRF-receptor 1 blockade attenuates acute posttraumatic hyperglycemia in rats. J. Surg. Res. 119: 72-79.

Hoshino M, Haraguchi Y, Mizushima I and Sakai M (2010). Close relationship between strict blood glucose control, including suppression of blood glucose variability, and mortality reduction in acutely ill patients with glucose intolerance investigated by means of a bedside-type artificial pancreas. J. Artif. Organs 13: 151-160.

Ichiki H, Hamasaki S, Nakasaki M, Ishida S, et al. (2010). Relationship between hyperglycemia and coronary vascular resistance in non-diabetic patients. Int. J. Cardiol. 141: 44-48.

Kimura K (2005). Biological effects of proinsulin C-peptide. Seikagakv 77: 419-423.

Knaus WA, Draper EA, Wagner DP and Zimmerman JE (1985). APACHE II: a severity of disease classification system. Crit. Care Med. 13: 818-829.

Leonidou L, Mouzaki A, Michalaki M, DeLastic AL, et al. (2007). Cytokine production and hospital mortality in patients with sepsis-induced stress hyperglycemia. J. Infect. 55: 340-346.

Marette A (2002). Mediators of cytokine-induced insulin resistance in obesity and other inflammatory settings. Curr. Opin. Clin. Nutr. Metab. Care 5: 377-383.

McCowen KC, Malhotra A and Bistrian BR (2001). Stress-induced hyperglycemia. Crit. Care Clin. 17: 107-124.

McMahon MM and Bistrian BR (1995). Host defenses and susceptibility to infection in patients with diabetes mellitus. Infect. Dis. Clin. North Am. 9: 1-9.

Nakamura T, Ako J, Kadowaki T, Funayama H, et al. (2009). Impact of acute hyperglycemia during primary stent implantation in patients with ST-elevation myocardial infarction. J. Cardiol. 53: 272-277.

Norhammar A, Tenerz A, Nilsson G, Hamsten A, et al. (2002). Glucose metabolism in patients with acute myocardial infarction and no previous diagnosis of diabetes mellitus: a prospective study. Lancet 359: 2140-2144.

Selvin E, Marinopoulos S, Berkenblit G, Rami T, et al. (2004). Meta-analysis: glycosylated hemoglobin and cardiovascular disease in diabetes mellitus. Ann. Intern. Med. 141: 421-431. 
Umpierrez GE, Isaacs SD, Bazargan N, You X, et al. (2002). Hyperglycemia: an independent marker of in-hospital mortality in patients with undiagnosed diabetes. J. Clin. Endocrinol. Metab. 87: 978-982.

van den Berghe G, Wouters P, Weekers F, Verwaest C, et al. (2001). Intensive insulin therapy in critically ill patients. $N$. Engl. J. Med. 345: 1359-1367. 